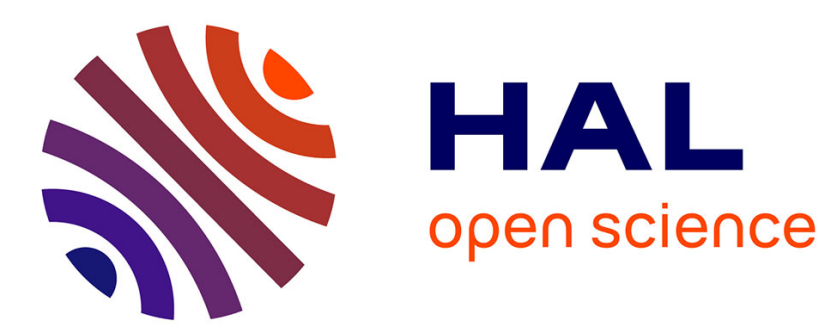

\title{
Cross-sections and rate coefficients for rotational excitation of aluminium hydroxide by helium
}

\author{
Manel Naouai, Faouzi Najar, Cheikh Bop, Kamel Hammami
}

\section{To cite this version:}

Manel Naouai, Faouzi Najar, Cheikh Bop, Kamel Hammami. Cross-sections and rate coefficients for rotational excitation of aluminium hydroxide by helium. Monthly Notices of the Royal Astronomical Society, 2018, 480 (4), pp.5412 - 5418. 10.1093/mnras/sty2223 . hal-03078747

\section{HAL Id: hal-03078747 https://hal.science/hal-03078747}

Submitted on 16 Dec 2020

HAL is a multi-disciplinary open access archive for the deposit and dissemination of scientific research documents, whether they are published or not. The documents may come from teaching and research institutions in France or abroad, or from public or private research centers.
L'archive ouverte pluridisciplinaire HAL, est destinée au dépôt et à la diffusion de documents scientifiques de niveau recherche, publiés ou non, émanant des établissements d'enseignement et de recherche français ou étrangers, des laboratoires publics ou privés. 


\title{
Cross-sections and rate coefficients for rotational excitation of aluminium hydroxide by helium
}

\author{
Manel Naouai (D), ${ }^{1 \star}$ Faouzi Najar, ${ }^{1}$ Cheikh Tidiane Bop (D) ${ }^{2}$ and Kamel Hammami ${ }^{1}$ \\ ${ }^{1}$ Laboratoire de Spectroscopie Atomique Moléculaire et Applications, Département de Physique, Faculté des Sciences de Tunis, Campus Universitaire Farhat \\ Hached, 2092 Tunis ElManar, Tunisia \\ ${ }^{2}$ Laboratoire Atomes Lasers, Département de Physique, Faculté des Sciences et Techniques, Université Cheikh Anta Diop, Dakar 5005, Senegal
}

Accepted 2018 August 13. Received 2018 June 30; in original form 2018 February 15

\begin{abstract}
Aluminium hydroxide $(\mathrm{AlOH})$ is ubiquitous in the interstellar medium. However, there are no reliable data available in the literature that can be used to interpret the observations. Therefore, in this paper, we deal with the rotational rate coefficients of $\mathrm{AlOH}$ induced by collision with helium. The potential energy surface of the $\mathrm{AlOH}-\mathrm{He}$ complex was computed within the coupled cluster ab initio method with single, double and perturbative triple excitation, in conjunction with the augmented-correlation consistent-polarized valence quadruple zeta Gaussian basis set. Using this potential energy surface, we computed rotational cross-sections for total energies ranging up to $1500 \mathrm{~cm}^{-1}$ with the close-coupling approach. By thermally averaging these cross-sections over the kinetic temperature range 5-300 K, we derived the $\mathrm{AlOH}$ rate coefficients corresponding to the first 13 rotational levels. Propensity rules have been found in favour of $|\Delta j|=1$. The data calculated in this paper have been compared with previous results and significant differences are observed. Such a finding might greatly affect the calculation of the $\mathrm{AlOH}$ abundance. In addition, this might encourage experimental work to settle the matter.
\end{abstract}

Key words: molecular data-molecular processes-scattering-ISM: abundances-ISM: molecules.

\section{INTRODUCTION}

A large number of aluminium-containing species has been detected in the interstellar medium (ISM). Indeed, aluminium isocyanide (AlCN; Ziurys et al. 2002), aluminium fluoride (AlF) and aluminium chloride $(\mathrm{AlCl})$ were discovered toward the asymptotic giant branch star IRC+10216 in the envelopes of carbon-rich evolved stars (Ziurys 2006).

Because of the high abundance of its elemental form, aluminium is thought to be the main precursor of $\mathrm{AlOH}$ through the single replacement reaction ( Morton et al. 1973)

$\mathrm{Al}+\mathrm{H}_{2} \mathrm{O} \longrightarrow \mathrm{AlOH}+\mathrm{H}$.

This chemical equation has been the subject of several investigations, theoretical as well as experimental (Hauge, Kauffman \& Margrave 1980; McClean, Nelson \& Campbell 1993; Álvarez-Barcia $\&$ Flores 2011, 2016). These studies provide some additional information: the chemical reaction in equation (1) is favourable in the gas phase.

In radio astronomy, $\mathrm{AlOH}$ is considered to be one of the most important aluminium-bearing species because of its large electric

^E-mail: manelnaouai6@gmail.com dipole moment (Li et al. 2003). Recently, along with several other Al-bearing molecules, $\mathrm{AlOH}$ was identified towards the circumstellar gas of Mira (o Ceti) by Kamiński et al. (2016). Using the Atacama Pathfinder Experiment, they found three rotational transitions of AlOH: $13 \rightarrow 12,15 \rightarrow 14$ and $35 \rightarrow 34$. In addition, this molecule was observed towards the envelope of the oxygen-rich star, VY Canis Majoris (VY CMa), using the Arizona Radio Observatory (ARO; Tenenbaum \& Ziurys 2010). More precisely, three rotational emission lines were detected: $9 \rightarrow 8,7 \rightarrow 6$ and $5 \rightarrow 4$. It is worth noting that $\mathrm{AlO}$, which is the second aluminium reservoir in VY CMa with a fractional abundance $\sim 17$ times smaller than that of $\mathrm{AlOH}$, was also observed with the ARO (Tenenbaum \& Ziurys 2009). Therefore, AlOH plays an important role in the chemistry of aluminium in the super-giant VY CMa star, making it crucial to determine its exact abundance in this medium. In fact, its abundance has been estimated at $\sim 1 \times 10^{-7}$ based on radiative transfer analysis. In addition, assuming the local thermodynamic equilibrium (LTE) model of Tsuji (1973), Tenenbaum \& Ziurys (2010) predicted an abundance of $\sim 2 \times 10^{-6}$.

To satisfy the astrophysical precision when modelling the interstellar molecular clouds, radiative transitions and rate coefficients induced by the most abundant collision partners (such as $\mathrm{He}$ and $\mathrm{H}_{2}$ ) are necessary. For $\mathrm{AlOH}$, rate coefficients induced by collision with 


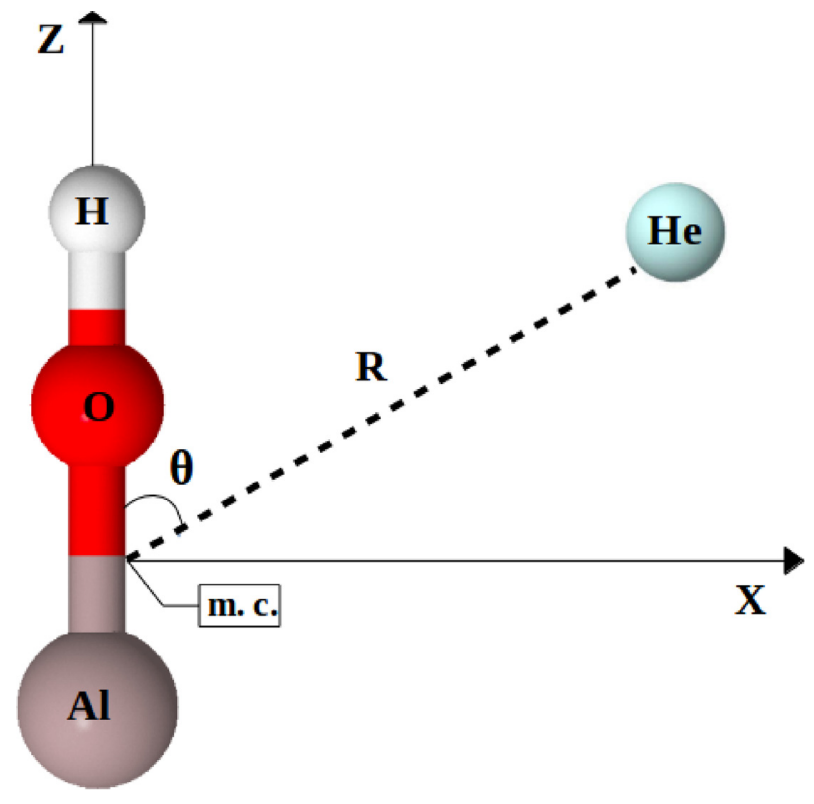

Figure 1. Jacobi coordinate system $(R, \theta)$ of $\mathrm{AlOH}-\mathrm{He}$.

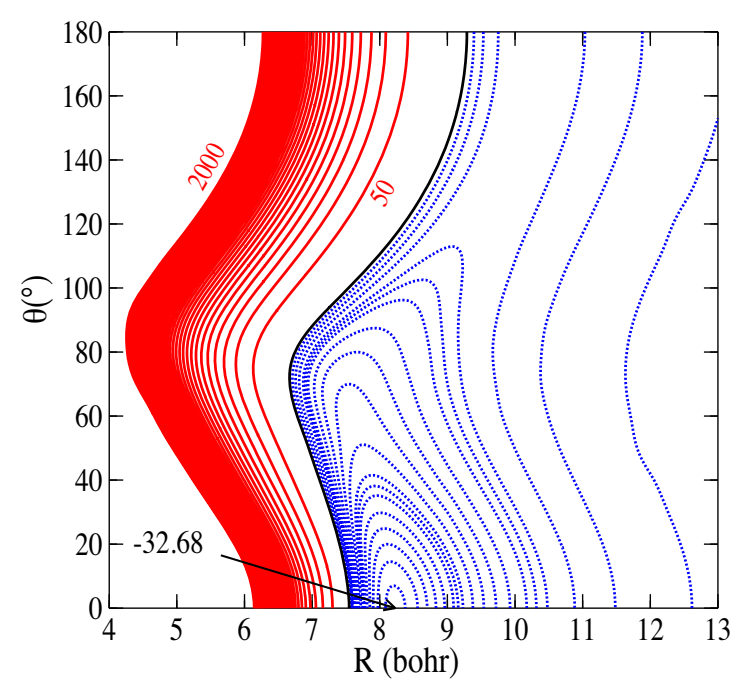

Figure 2. Contour plot of the interaction energy (in $\mathrm{cm}^{-1}$ ). Negative contour lines (blue) are equally spaced by $2 \mathrm{~cm}^{-1}$ and positive contour lines (red) are equally spaced by $50 \mathrm{~cm}^{-1}$. The zero energy contour line is black.

He have been reported by Tchakoua et al. (2015). However, these data have not been without criticism. In terms of magnitude rank, the rate coefficients of the $\mathrm{AlOH}-\mathrm{He}$ system computed by Tchakoua et al. (2015) are much larger than those of similar systems such as $\mathrm{HCP}-\mathrm{He}$ and $\mathrm{AlF}-\mathrm{He}$ (for further discussion, see Section 4). In this regime, it is necessary to recompute the collisional rates of $\mathrm{AlOH}$ due to He impact, in order to provide reliable astrophysical data.

Although the $\mathrm{AlOH}$ emission lines observed in the ISM are rotational, theoretical calculations cannot be performed regardless of the vibrational modes. For this purpose, the $\mathrm{AlOH}$ vibrational frequencies should be determined first. Indeed, these have been the target of various experimental and theoretical studies. For example, Pilgrim, Robbins \& Duncan (1993) measured the Al-OH stretch frequency $\left(v_{\mathrm{Al}-\mathrm{OH}}=895 \mathrm{~cm}^{-1}\right)$ and Hauge et al. (1980) measured in a noble gas matrix the two stretch frequency modes $\left(v_{\mathrm{Al}-\mathrm{OH}}=\right.$

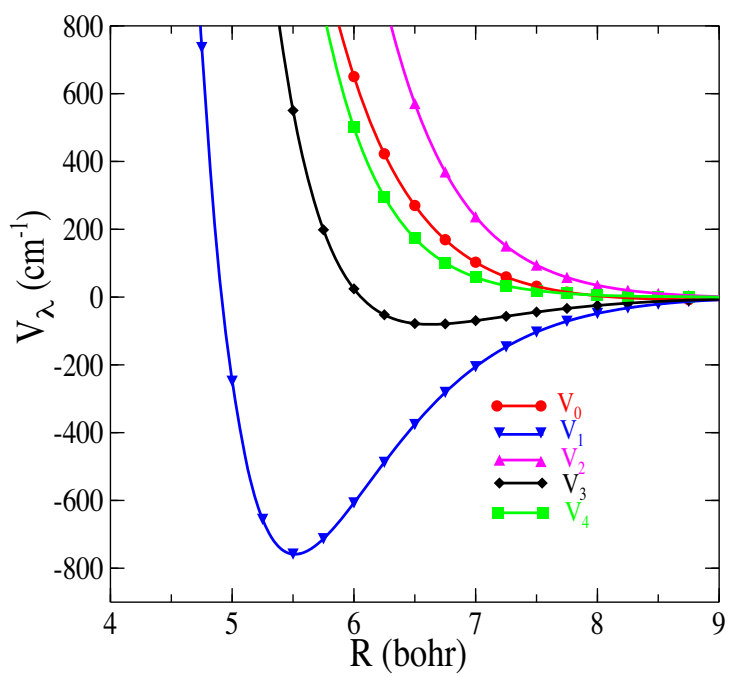

Figure 3. Radial coefficients $V_{\lambda}(\lambda=0, \ldots, 4)$ of the interaction energy as a function of the intermolecular distance $R$.

$810 \mathrm{~cm}^{-1}$ and $v_{\mathrm{AlO}-\mathrm{H}}=3790 \mathrm{~cm}^{-1}$ ). These measurements have been theoretically $\left(v_{\mathrm{Al}-\mathrm{OH}}=884 \mathrm{~cm}^{-1}\right.$ and $\left.v_{\mathrm{AlO}-\mathrm{H}}=4313 \mathrm{~cm}^{-1}\right)$ confirmed by Vacek, Deleeuw \& Schaefer (1993).

This paper is organized as follows. In Section 2, we describe the new potential energy surface (PES) of $\mathrm{AlOH}-\mathrm{He}$ as well as the computational details. In Section 3, we outline the inelastic cross-sections and rate coefficients of the AlOH-He complex. In Section 4, we present a comparative study between the present work and the previous work. Finally, we give concluding remarks in Section 5.

\section{POTENTIAL ENERGY SURFACE}

The studied system is made up of a linear molecule $(\mathrm{AlOH})$ and a structureless atom $(\mathrm{He})$. In the rigid rotator approximation, the $\mathrm{Al}-\mathrm{O}$ and $\mathrm{O}-\mathrm{H}$ bond lengths are frozen into their experimental equilibrium geometries, $r_{\mathrm{AlO}}=3.1796 \$ \mathrm{bohr}$ and $r_{\mathrm{OH}}=1.6597$ bohr (Apponi, Barclay \& Ziurys 1993). As illustrated in Fig. 1, the complex is described by the Jacobi coordinate system $(R, \theta)$, where $R$ is the intermolecular distance and $\theta$ represents the enclosed angle between the intermolecular axis and the $\mathrm{AlOH}$ axis. The $\mathrm{AlOH}-\mathrm{He}$ van der Waals system can be described using one single electron configuration. For this purpose, we performed preliminary calculations using the complete active space self-consistent field (CASSCF) approach to check the validity of this approximation (Knowles \& Werner 1985; Werner \& Knowles 1985). Indeed, the dominant configuration of the ground electronic state weighs $\sim 0.96$ for all geometries. Therefore, the coupled cluster method with single, double and perturbative triple excitation, hereafter CCSD(T) (Adlerc, Knizia \& Werner 2007) is suitable to probe the interaction potential of this monoconfigurational system. This approach supplemented by the augmented-correlation consistent-polarized Valence Quadruple Zeta (aug-cc-pVQZ) basis set (Kendall, Dunning $\&$ Harrison 1992) and mid-bond functions of Cybulski \& Toczylowski (1999) [3s 3p 2d2f1g] - hereafter, this level of theory will be denoted as CCSD(T)/aug-cc-pVQZ+bf - was employed as implemented in the MOLPRO-2010 quantum chemistry package (Werner et al. 2010) to determine the interaction energy. This latter was calculated from $R=4-100$ bohr $(4,4.25,4.5,4.75,5,5.25,5.5,5.75$, $6,6.25,6.5,6.75,7,7.25,7.5,7.75,8,8.25,8.5,8.75,9,9.25,9.5$, 
Table 1. MOLSCAT parameter values.

\begin{tabular}{|c|c|c|c|c|c|}
\hline$R_{\min }(\mathrm{bohr})$ & $R_{\max }(\mathrm{bohr})$ & STEPS & $\mu(\mathrm{au})$ & $B_{0}\left(\mathrm{~cm}^{-1}\right)$ & $D_{0}\left(\mathrm{~cm}^{-1}\right)$ \\
\hline 3 & 30 & 20,16 & & & \\
\hline
\end{tabular}

${ }^{a}$ See Apponi et al. (1993).

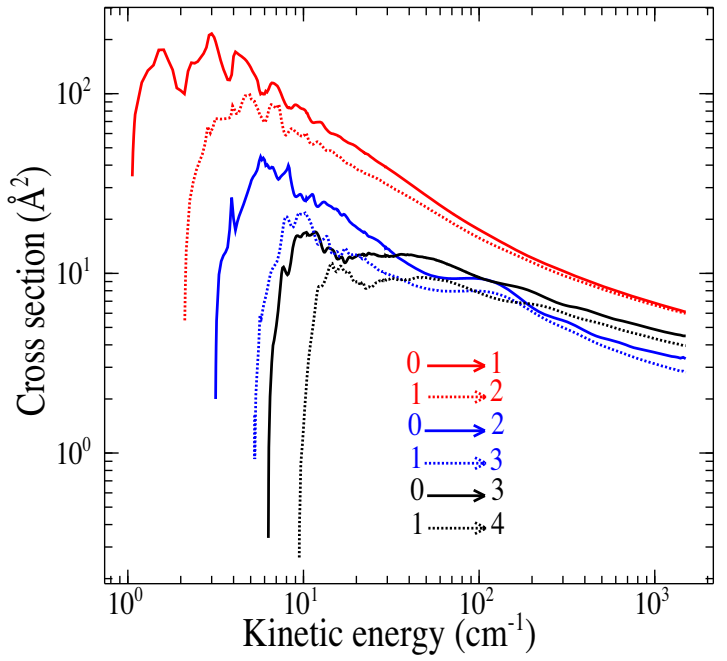

Figure 4. Rotational excitation cross-sections of $\mathrm{AlOH}$ by $\mathrm{He}$ as a function of the kinetic energy for $\Delta j=1,2,3$ transitions.

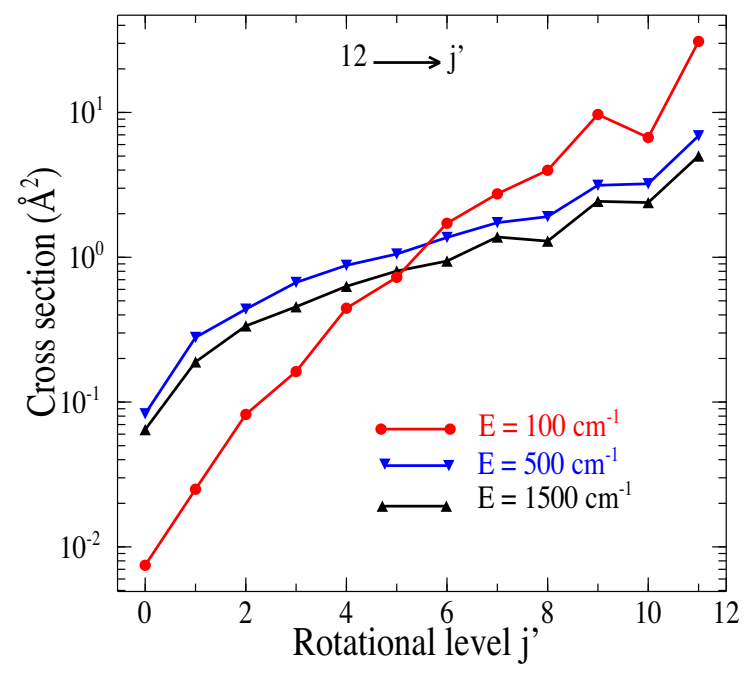

Figure 5. De-excitation cross-sections at total energies $E=100,500$ and $1500 \mathrm{~cm}^{-1}$ from the rotational level $j=12$ to $j^{\prime}=11, \ldots, 0$.

$9.75,10,10.5,11,11.5,12,12.5,13,13.5,14,14.5,15,15.5,16$ $16.5,17,17.5,18,18.5,19,19.5,20,22,24,26,28,30,32,34$, $36,38,40,50,100)$ and $\theta$ from $0^{\circ}$ to $180^{\circ}$ with a step of $10^{\circ}$. It is worth mentioning that the basis set superposition error (BSSE) was corrected using the counterpoise procedure of Boys \& Bernardi (1970),

$V(R, \theta)=E_{\mathrm{AlOH}-\mathrm{He}}(R, \theta)-E_{\mathrm{AlOH}}(R, \theta)-E_{\mathrm{He}}(R, \theta)$,

where $E_{\mathrm{AlOH}-\mathrm{He}}$ is the energy of the complex and $E_{\mathrm{AlOH}}$ and $E_{\mathrm{He}}$ are calculated in the full basis set of the complex stand for the energies of $\mathrm{AlOH}$ and $\mathrm{He}$, respectively.

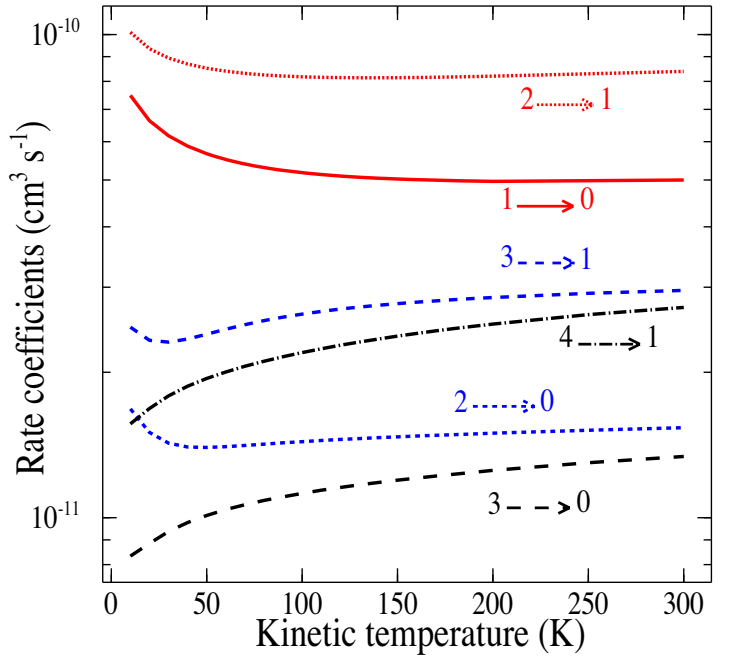

Figure 6. Rotational de-excitation rate coefficients of $\mathrm{AlOH}$ by $\mathrm{He}$ as a function of kinetic temperature for $\Delta j=-1,-2,-3$ transitions.

Fig. 2 illustrates some contour plots of the AlOH-He PES. As can be seen, the interaction potential presents a strong anisotropy with a single well depth of $-32.680 \mathrm{~cm}^{-1}$ located at $\theta=0^{\circ}$ and $R=$ 8.2 bohr (i.e. towards the $\mathrm{H}$ end of the $\mathrm{AlOH}$ molecule). In addition, the shape is typically identical to the PES of Tchakoua et al. (2015), which has a slightly deeper potential well $\left(-32.995 \mathrm{~cm}^{-1}\right)$. Hence, our PES can be unambiguously used to perform the dynamical calculations.

We have expanded the PES over the Legendre polynomial functions to determine the basic input needed in the dynamic computations :

$V(R, \theta)=\sum_{\lambda=0}^{\lambda_{\max }=18} V_{\lambda}(R) P_{\lambda}(\cos \theta)$.

We included 19 radial terms $\left(V_{\lambda}\right)$ in the development and this led to an analytical PES similar to the $a b$ initio PES with deviations of a few $\mathrm{cm}^{-1}$. Fig. 3 illustrates the $R$ dependence of the first five $V_{\lambda}$. The radial coefficients corresponding to $\lambda=1$ strongly outweigh the others. The impact of such a predominance on the cross-sections and rate coefficients is discussed in the next section.

\section{DYNAMICAL STUDY}

\subsection{Cross-sections}

We computed the rotational energy levels of $\mathrm{AlOH}$ using the experimental spectroscopic constants presented in Table 1. The rotational cross-sections of $\mathrm{AlOH}$ in collision with He were calculated using the close coupling (CC) approach (Arthurs \& Dalgarno 1960) as is implemented in the MOLSCAT quantum package (Hutson \& Green 1994). It is worth mentioning that the coupled equations have been 
Table 2. Rotational de-excitation rate coefficients of $\mathrm{AlOH}$ by He as a function of kinetic temperature (in $\mathrm{K}$ ) for $1 \leq j \leq 12$.

\begin{tabular}{|c|c|c|c|c|c|c|c|c|c|c|}
\hline \multirow[t]{2}{*}{$j$} & \multirow[t]{2}{*}{$j^{\prime}$} & \multicolumn{9}{|c|}{$K_{j \rightarrow j^{\prime}}\left(\mathrm{cm}^{3} \mathrm{~s}^{-1}\right)$} \\
\hline & & 5 & 10 & 30 & 50 & 100 & 150 & 200 & 250 & 300 \\
\hline 1 & 0 & $8.3164(-11)$ & $7.4824(-11)$ & $6.1724(-11)$ & $5.6672(-11)$ & $5.1772(-11)$ & $5.0243(-11)$ & $4.9812(-11)$ & $4.9828(-11)$ & $4.9999(-11)$ \\
\hline 2 & 0 & $1.8117(-11)$ & $1.6798(-11)$ & $1.4270(-11)$ & $1.3975(-11)$ & $1.4371(-11)$ & $1.4703(-11)$ & $1.4955(-11)$ & $1.5172(-11)$ & $1.5357(-11)$ \\
\hline 2 & 1 & $1.0745(-10)$ & $1.0107(-10)$ & $8.9409(-11)$ & $8.5171(-11)$ & $8.1753(-11)$ & $8.1408(-11)$ & $8.2009(-11)$ & $8.2935(-11)$ & $8.3881(-11)$ \\
\hline 3 & 0 & $8.2213(-12)$ & $8.3295(-12)$ & $9.3635(-12)$ & $1.0112(-11)$ & $1.1233(-11)$ & $1.1960(-11)$ & $1.2527(-11)$ & $1.2997(-11)$ & $1.3385(-11)$ \\
\hline 3 & 1 & $2.5947(-11)$ & $2.4799(-11)$ & $2.3090(-11)$ & $2.3981(-11)$ & $2.6391(-11)$ & $2.7741(-11)$ & $2.8565(-11)$ & $2.9138(-11)$ & $2.9551(-11)$ \\
\hline 3 & 2 & $1.1334(-10)$ & $1.0979(-10)$ & $9.7555(-11)$ & $9.3263(-11)$ & $9.0503(-11)$ & $9.0961(-11)$ & $9.2256(-11)$ & $9.3756(-11)$ & $9.5160(-11)$ \\
\hline 4 & 0 & $2.6605(-12)$ & $2.6693(-12)$ & $3.2133(-12)$ & $3.8145(-12)$ & $4.7875(-12)$ & $5.2719(-12)$ & $5.5335(-12)$ & $5.6861(-12)$ & $5.7755(-12)$ \\
\hline 4 & 1 & $1.4609(-11)$ & $1.5643(-11)$ & $1.7870(-11)$ & $1.9421(-11)$ & $2.1966(-11)$ & $2.3764(-11)$ & $2.5173(-11)$ & $2.6322(-11)$ & $2.7254(-11)$ \\
\hline 4 & 2 & $2.9401(-11)$ & $2.9108(-11)$ & $2.7404(-11)$ & $2.9175(-11)$ & $3.3523(-11)$ & $3.5949(-11)$ & $3.7392(-11)$ & $3.8353(-11)$ & $3.9016(-11)$ \\
\hline 4 & 3 & $1.0993(-10)$ & $1.1106(-10)$ & $1.0011(-10)$ & $9.5608(-11)$ & $9.2756(-11)$ & $9.3477(-11)$ & $9.5130(-11)$ & $9.6974(-11)$ & $9.8674(-11)$ \\
\hline 5 & 0 & $2.0381(-12)$ & $2.2244(-12)$ & $2.8649(-12)$ & $3.3070(-12)$ & $4.0563(-12)$ & $4.6016(-12)$ & $5.0189(-12)$ & $5.3472(-12)$ & $5.6060(-12)$ \\
\hline 5 & 1 & $5.3788(-12)$ & $5.5877(-12)$ & $7.0973(-12)$ & $8.8652(-12)$ & $1.1846(-11)$ & $1.3354(-11)$ & $1.4176(-11)$ & $1.4661(-11)$ & $1.4952(-11)$ \\
\hline 5 & 2 & $1.7537(-11)$ & $1.9100(-11)$ & $2.1465(-11)$ & $2.3055(-11)$ & $2.5893(-11)$ & $2.8141(-11)$ & $3.0012(-11)$ & $3.1574(-11)$ & $3.2854(-11)$ \\
\hline 5 & 3 & $2.9430(-11)$ & $2.9720(-11)$ & $2.8567(-11)$ & $3.0843(-11)$ & $3.6654(-11)$ & $4.0174(-11)$ & $4.2357(-11)$ & $4.3831(-11)$ & $4.4856(-11)$ \\
\hline 5 & 4 & $1.0875(-10)$ & $1.1128(-10)$ & $1.0133(-10)$ & $9.6802(-11)$ & $9.3987(-11)$ & $9.4861(-11)$ & $9.6719(-11)$ & $9.8773(-11)$ & $1.0066(-10)$ \\
\hline 6 & 0 & $8.9361(-13)$ & $9.5684(-13)$ & $1.5509(-12)$ & $2.2023(-12)$ & $3.2646(-12)$ & $3.7905(-12)$ & $4.0752(-12)$ & $4.2429(-12)$ & $4.3442(-12)$ \\
\hline 6 & 1 & $4.2695(-12)$ & $4.3961(-12)$ & $5.4528(-12)$ & $6.3024(-12)$ & $7.8398(-12)$ & $9.0812(-12)$ & $1.0095(-11)$ & $1.0916(-11)$ & $1.1574(-11)$ \\
\hline 6 & 2 & $7.0488(-12)$ & $7.2174(-12)$ & $8.9656(-12)$ & $1.1278(-11)$ & $1.5712(-11)$ & $1.8220(-11)$ & $1.9684(-11)$ & $2.0595(-11)$ & $2.1175(-11)$ \\
\hline 6 & 3 & $2.1016(-11)$ & $2.2034(-11)$ & $2.3963(-11)$ & $2.5392(-11)$ & $2.8257(-11)$ & $3.0702(-11)$ & $3.2818(-11)$ & $3.4624(-11)$ & $3.6119(-11)$ \\
\hline 6 & 4 & $2.9502(-11)$ & $2.9520(-11)$ & $2.8657(-11)$ & $3.1111(-11)$ & $3.7402(-11)$ & $4.1477(-11)$ & $4.4154(-11)$ & $4.6032(-11)$ & $4.7379(-11)$ \\
\hline 6 & 5 & $1.1116(-10)$ & $1.1265(-10)$ & $1.0253(-10)$ & $9.7852(-11)$ & $9.5074(-11)$ & $9.6181(-11)$ & $9.8289(-11)$ & $1.0057(-10)$ & $1.0264(-10)$ \\
\hline 7 & 1 & $1.9531(-12)$ & $2.0364(-12)$ & $3.2599(-12)$ & $4.7713(-12)$ & $7.5876(-12)$ & $9.1621(-12)$ & $1.0080(-11)$ & $1.0653(-11)$ & $1.1021(-11)$ \\
\hline 7 & 2 & $5.8507(-12)$ & $5.7600(-12)$ & $6.7505(-12)$ & $7.7333(-12)$ & $9.6935(-12)$ & $1.1347(-11)$ & $1.2738(-11)$ & $1.3888(-11)$ & $1.4819(-11)$ \\
\hline 7 & 3 & $8.8562(-12)$ & $8.7511(-12)$ & $1.0276(-11)$ & $1.2596(-11)$ & $1.7439(-11)$ & $2.0454(-11)$ & $2.2351(-11)$ & $2.3606(-11)$ & $2.4452(-11)$ \\
\hline 7 & 4 & $2.5475(-11)$ & $2.5374(-11)$ & $2.6286(-11)$ & $2.7389(-11)$ & $3.0178(-11)$ & $3.2827(-11)$ & $3.5184(-11)$ & $3.7213(-11)$ & $3.8899(-11)$ \\
\hline 7 & 5 & $2.9723(-11)$ & $2.9326(-11)$ & $2.8528(-11)$ & $3.1094(-11)$ & $3.7414(-11)$ & $4.1562(-11)$ & $4.4381(-11)$ & $4.6424(-11)$ & $4.7934(-11)$ \\
\hline 7 & 6 & $1.1438(-10)$ & $1.1437(-10)$ & $1.0367(-10)$ & $9.8796(-11)$ & $9.5894(-11)$ & $9.7158(-11)$ & $9.9504(-11)$ & $1.0202(-10)$ & $1.0431(-10)$ \\
\hline 8 & 2 & $2.9185(-12)$ & $2.8984(-12)$ & $4.2724(-12)$ & $6.0858(-12)$ & $9.7160(-12)$ & $1.1951(-11)$ & $1.3358(-11)$ & $1.4291(-11)$ & $1.4925(-11)$ \\
\hline 8 & 3 & $7.6550(-12)$ & $7.1634(-12)$ & $7.8043(-12)$ & $8.7546(-12)$ & $1.1002(-11)$ & $1.3038(-11)$ & $1.4774(-11)$ & $1.6216(-11)$ & $1.7385(-11)$ \\
\hline 8 & 4 & $1.1158(-11)$ & $1.0530(-11)$ & $1.1573(-11)$ & $1.3728(-11)$ & $1.8402(-11)$ & $2.1441(-11)$ & $2.3443(-11)$ & $2.4829(-11)$ & $2.5804(-11)$ \\
\hline 8 & 5 & $3.1030(-11)$ & $2.9288(-11)$ & $2.8631(-11)$ & $2.9260(-11)$ & $3.1728(-11)$ & $3.4477(-11)$ & $3.7044(-11)$ & $3.9295(-11)$ & $4.1180(-11)$ \\
\hline 8 & 6 & $3.0357(-11)$ & $2.9395(-11)$ & $2.8420(-11)$ & $3.1072(-11)$ & $3.7387(-11)$ & $4.1471(-11)$ & $4.4260(-11)$ & $4.6313(-11)$ & $4.7854(-11)$ \\
\hline 8 & 7 & $1.1890(-10)$ & $1.1658(-10)$ & $1.0481(-10)$ & $9.9713(-11)$ & $9.6556(-11)$ & $9.7784(-11)$ & $1.0022(-10)$ & $1.0288(-10)$ & $1.0531(-10)$ \\
\hline 9 & 3 & $3.7854(-12)$ & $3.7537(-12)$ & $5.1770(-12)$ & $7.0746(-12)$ & $1.0899(-11)$ & $1.3336(-11)$ & $1.4942(-11)$ & $1.6058(-11)$ & $1.6849(-11)$ \\
\hline 9 & 4 & $8.9402(-12)$ & $8.3989(-12)$ & $8.7447(-12)$ & $9.5846(-12)$ & $1.1913(-11)$ & $1.4239(-11)$ & $1.6293(-11)$ & $1.8023(-11)$ & $1.9435(-11)$ \\
\hline 9 & 5 & $1.2897(-11)$ & $1.2184(-11)$ & $1.2865(-11)$ & $1.4854(-11)$ & $1.9262(-11)$ & $2.2145(-11)$ & $2.4071(-11)$ & $2.5435(-11)$ & $2.6417(-11)$ \\
\hline 9 & 6 & $3.4635(-11)$ & $3.2625(-11)$ & $3.0806(-11)$ & $3.0978(-11)$ & $3.2994(-11)$ & $3.5660(-11)$ & $3.8302(-11)$ & $4.0689(-11)$ & $4.2719(-11)$ \\
\hline 9 & 7 & $3.0003(-11)$ & $2.9237(-11)$ & $2.8314(-11)$ & $3.1061(-11)$ & $3.7424(-11)$ & $4.1478(-11)$ & $4.4240(-11)$ & $4.6279(-11)$ & $4.7818(-11)$ \\
\hline 9 & 8 & $1.1904(-10)$ & $1.1723(-10)$ & $1.0556(-10)$ & $1.0043(-10)$ & $9.7119(-11)$ & $9.8224(-11)$ & $1.0061(-10)$ & $1.0328(-10)$ & $1.0575(-10)$ \\
\hline 10 & 4 & $4.8842(-12)$ & $4.7540(-12)$ & $6.1297(-12)$ & $8.0466(-12)$ & $1.1864(-11)$ & $1.4287(-11)$ & $1.5903(-11)$ & $1.7050(-11)$ & $1.7883(-11)$ \\
\hline 10 & 5 & $1.0464(-11)$ & $9.7282(-12)$ & $9.6654(-12)$ & $1.0351(-11)$ & $1.2584(-11)$ & $1.5013(-11)$ & $1.7255(-11)$ & $1.9191(-11)$ & $2.0797(-11)$ \\
\hline 10 & 6 & $1.5043(-11)$ & $1.4030(-11)$ & $1.4215(-11)$ & $1.6020(-11)$ & $2.0160(-11)$ & $2.2880(-11)$ & $2.4706(-11)$ & $2.6008(-11)$ & $2.6954(-11)$ \\
\hline 10 & 7 & $3.8822(-11)$ & $3.6186(-11)$ & $3.2990(-11)$ & $3.2666(-11)$ & $3.4170(-11)$ & $3.6621(-11)$ & $3.9185(-11)$ & $4.1572(-11)$ & $4.3641(-11)$ \\
\hline 10 & 8 & $3.0241(-11)$ & $2.9402(-11)$ & $2.8341(-11)$ & $3.1114(-11)$ & $3.7509(-11)$ & $4.1557(-11)$ & $4.4321(-11)$ & $4.6370(-11)$ & $4.7923(-11)$ \\
\hline 10 & 9 & $1.2098(-10)$ & $1.1853(-10)$ & $1.0641(-10)$ & $1.0118(-10)$ & $9.7713(-11)$ & $9.8672(-11)$ & $1.0094(-10)$ & $1.0353(-10)$ & $1.0594(-10)$ \\
\hline 11 & 5 & $5.9840(-12)$ & $5.8018(-12)$ & $7.1134(-12)$ & $9.0277(-12)$ & $1.2796(-11)$ & $1.5166(-11)$ & $1.6747(-11)$ & $1.7876(-11)$ & $1.8705(-11)$ \\
\hline 11 & 6 & $1.1688(-11)$ & $1.0922(-11)$ & $1.0526(-11)$ & $1.1060(-11)$ & $1.3145(-11)$ & $1.5544(-11)$ & $1.7828(-11)$ & $1.9848(-11)$ & $2.1551(-11)$ \\
\hline 11 & 7 & $1.6906(-11)$ & $1.5768(-11)$ & $1.5528(-11)$ & $1.7159(-11)$ & $2.1059(-11)$ & $2.3639(-11)$ & $2.5385(-11)$ & $2.6642(-11)$ & $2.7564(-11)$ \\
\hline 11 & 8 & $4.1796(-11)$ & $3.9167(-11)$ & $3.4993(-11)$ & $3.4227(-11)$ & $3.5259(-11)$ & $3.7474(-11)$ & $3.9893(-11)$ & $4.2193(-11)$ & $4.4210(-11)$ \\
\hline 11 & 9 & $3.0245(-11)$ & $2.9530(-11)$ & $2.8432(-11)$ & $3.1202(-11)$ & $3.7611(-11)$ & $4.1657(-11)$ & $4.4429(-11)$ & $4.6498(-11)$ & $4.8076(-11)$ \\
\hline 11 & 10 & $1.2135(-10)$ & $1.1915(-10)$ & $1.0705(-10)$ & $1.0181(-10)$ & $9.8265(-11)$ & $9.9121(-11)$ & $1.0128(-10)$ & $1.0376(-10)$ & $1.0608(-10)$ \\
\hline 12 & 6 & $7.1053(-12)$ & $6.8826(-12)$ & $8.1122(-12)$ & $1.0011(-11)$ & $1.3711(-11)$ & $1.6020(-11)$ & $1.7567(-11)$ & $1.8683(-11)$ & $1.9507(-11)$ \\
\hline 12 & 7 & $1.2724(-11)$ & $1.1985(-11)$ & $1.1299(-11)$ & $1.1701(-11)$ & $1.3658(-11)$ & $1.5992(-11)$ & $1.8239(-11)$ & $2.0248(-11)$ & $2.1959(-11)$ \\
\hline 12 & 8 & $1.8629(-11)$ & $1.7414(-11)$ & $1.6798(-11)$ & $1.8264(-11)$ & $2.1930(-11)$ & $2.4370(-11)$ & $2.6038(-11)$ & $2.7257(-11)$ & $2.8162(-11)$ \\
\hline 12 & 9 & $4.3999(-11)$ & $4.1604(-11)$ & $3.6739(-11)$ & $3.5622(-11)$ & $3.6281(-11)$ & $3.8297(-11)$ & $4.0568(-11)$ & $4.2750(-11)$ & $4.4673(-11)$ \\
\hline 12 & 10 & $3.0301(-11)$ & $2.9692(-11)$ & $2.8557(-11)$ & $3.1293(-11)$ & $3.7696(-11)$ & $4.1739(-11)$ & $4.4511(-11)$ & $4.6590(-11)$ & $4.8182(-11)$ \\
\hline 12 & 11 & $1.2129(-10)$ & $1.1943(-10)$ & $1.0748(-10)$ & $1.0230(-10)$ & $9.8777(-11)$ & $9.9578(-11)$ & $1.0166(-10)$ & $1.0405(-10)$ & $1.0627(-10)$ \\
\hline
\end{tabular}

solved using the log-derivative propagator of Manolopoulos (1986). For a best compromise between result accuracy and computational time, we carried out preliminary calculations to fix the integration parameters of the propagator. In addition, Table 3 displays some convergence tests for the rotational basis, $j_{\max }$. As can be seen, the convergence is reached with $j_{\max }=23$, for a total energy (E) of $600 \mathrm{~cm}^{-1}$. Indeed, the cross-sections slightly vary $(0.002$ $\AA^{2}$ ) when passing from $j_{\max }=21$ to 23 . For the entire total en- 
Table 3. Cross-sections (in $\AA^{2}$ ) for different values of $j_{\max }$ for total energy $E=600 \mathrm{~cm}^{-1}$.

\begin{tabular}{|c|c|c|c|c|c|c|c|c|c|}
\hline \multirow[t]{3}{*}{ Transitions } & \multirow[t]{3}{*}{$E\left(\mathrm{~cm}^{-1}\right)$} & \multicolumn{8}{|c|}{$j_{\max }$} \\
\hline & & \multicolumn{4}{|c|}{ Tchakoua et al. (2015) } & \multicolumn{4}{|c|}{ This work } \\
\hline & & 17 & 19 & 21 & 23 & 17 & 19 & 21 & 23 \\
\hline $0 \rightarrow 1$ & 600 & 75.884 & 75.884 & 75.884 & 75.884 & 8.012 & 8.012 & 8.012 & 8.012 \\
\hline $0 \rightarrow 2$ & & 4.423 & 4.423 & 4.423 & 4.423 & 4.180 & 4.180 & 4.180 & 4.180 \\
\hline $1 \rightarrow 2$ & & 77.776 & 77.776 & 77.776 & 77.776 & 7.723 & 7.722 & 7.722 & 7.722 \\
\hline $2 \rightarrow 3$ & & 78.666 & 78.666 & 78.666 & 78.666 & 7.498 & 7.498 & 7.498 & 7.498 \\
\hline $5 \rightarrow 6$ & & 79.405 & 79.405 & 79.405 & 79.405 & 7.123 & 7.126 & 7.126 & 7.126 \\
\hline $5 \rightarrow 7$ & & 8.290 & 8.290 & 8.290 & 8.290 & 3.866 & 3.867 & 3.866 & 3.866 \\
\hline $6 \rightarrow 7$ & & 79.552 & 79.552 & 79.552 & 79.552 & 7.172 & 7.168 & 7.167 & 7.167 \\
\hline $8 \rightarrow 10$ & & 8.527 & 8.527 & 8.527 & 8.527 & 3.592 & 3.513 & 3.512 & 3.512 \\
\hline $11 \rightarrow 7$ & & - & - & - & - & 1.708 & 1.714 & 1.713 & 1.712 \\
\hline $11 \rightarrow 8$ & & - & - & - & - & 3.071 & 3.058 & 3.054 & 3.054 \\
\hline $11 \rightarrow 9$ & & - & - & - & - & 3.019 & 2.977 & 2.981 & 2.983 \\
\hline $11 \rightarrow 10$ & & - & - & - & - & 6.545 & 6.528 & 6.528 & 6.527 \\
\hline $12 \rightarrow 8$ & & - & - & - & - & 1.864 & 1.759 & 1.756 & 1.757 \\
\hline $12 \rightarrow 9$ & & - & - & - & - & 3.090 & 3.038 & 3.051 & 3.052 \\
\hline $12 \rightarrow 10$ & & - & - & - & - & 3.270 & 3.045 & 2.999 & 2.997 \\
\hline $12 \rightarrow 11$ & & - & - & - & - & 6.608 & 6.575 & 6.550 & 6.548 \\
\hline
\end{tabular}

Table 4. Comparison the AlOH-He, $\mathrm{HCP}-\mathrm{He}$ and AlF-He global minima (in units of $\mathrm{cm}^{-1}$ ).

\begin{tabular}{lcccc}
\hline & Coordinates & This work & Hammami et al. & Gotoum et al. \\
\hline$R$ (bohr) & $\theta\left({ }^{\circ}\right)$ & AlOH-He & HCP-He & AlF-He \\
\hline 8.20 & 0 & 32.680 & - & 22.018 \\
9.20 & 0 & - & - & - \\
7.75 & 0 & - & & 24.056 \\
\hline
\end{tabular}

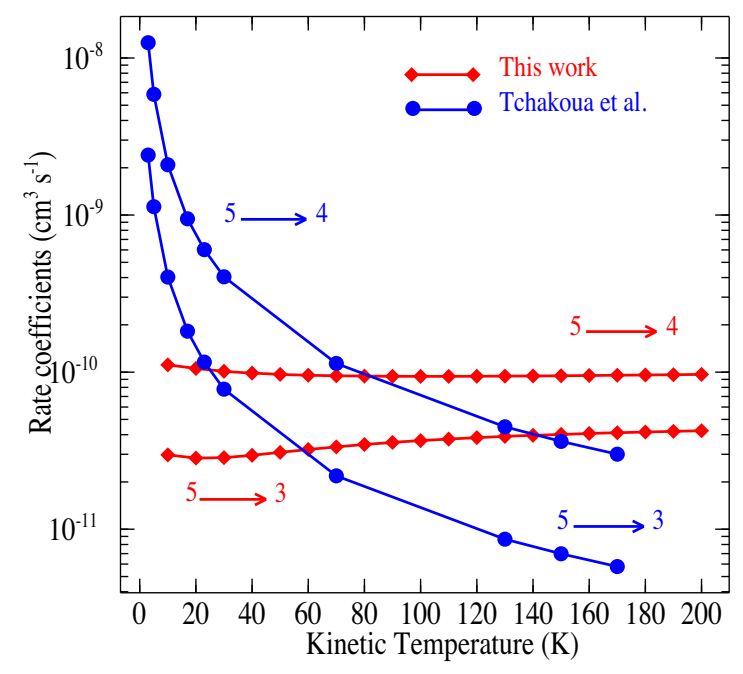

Figure 7. Our rotational de-excitation rate coefficients of $\mathrm{AlOH}-\mathrm{He}$ in comparison with results obtained by Tchakoua et al. (2015).

ergy range, which expands up to $1500 \mathrm{~cm}^{-1}, j_{\max }$ was fixed at 17 for $0 \leq E \leq 100 \mathrm{~cm}^{-1}, 20$ for $100 \leq E \leq 300 \mathrm{~cm}^{-1}, 23$ for $300 \leq E \leq 600 \mathrm{~cm}^{-1}, 27$ for $600 \leq E \leq 1000 \mathrm{~cm}^{-1}$ and 33 for $1000 \leq E \leq 1500 \mathrm{~cm}^{-1}$.

The total energy range was spanned using steps of $0.1 \mathrm{~cm}^{-1}$ for $0 \leq E \leq 35 \mathrm{~cm}^{-1}, 0.2 \mathrm{~cm}^{-1}$ for $35 \leq E \leq 100 \mathrm{~cm}^{-1}, 5 \mathrm{~cm}^{-1}$ for $100 \leq E \leq 300 \mathrm{~cm}^{-1}, 10 \mathrm{~cm}^{-1}$ for $300 \leq E \leq 600 \mathrm{~cm}^{-1}, 20 \mathrm{~cm}^{-1}$ for $600 \leq E \leq 1000 \mathrm{~cm}^{-1}$ and a step of $50 \mathrm{~cm}^{-1}$ for $1000 \leq E \leq$ $1500 \mathrm{~cm}^{-1}$.
Fig. 4 outlines the rotational excitation cross-sections for $\Delta j=$ 1,2 and 3. Several resonances are observed at low kinetic energies $\left(E_{k}\right)$, more precisely at $E_{k} \leq 30 \mathrm{~cm}^{-1}$. This behaviour is due to the global minimum of the interaction potential, which temporarily traps the He atom leading to the formation of quasi-bound states of the $\mathrm{AlOH}-\mathrm{He}$ complex. In addition, Fig. 4 shows that the $0 \rightarrow 1$ and $1 \rightarrow 2$ transitions outweigh in the whole kinetic energy range. Although the $\Delta j=2(0 \rightarrow 2$ and $1 \rightarrow 3)$ transitions predominate the $\Delta j=3$ transitions $(0 \rightarrow 3$ and $1 \rightarrow 4)$ at low kinetic energy, an inversion occurs at $E_{k} \geq 500 \mathrm{~cm}^{-1}$. Therefore, we can conclude that the propensity rules are in favour of the odd $\Delta j$ transitions. Indeed, this trend was predicted by the dominance of the $V_{1}$ and $V_{3}$ radial coefficients (see Fig. 3).

Fig. 5 displays the dependence of the de-excitation cross-sections with respect to the final rotational level (j') for selected kinetic energies $\left(E_{k}=100,500\right.$ and $\left.1500 \mathrm{~cm}^{-1}\right)$. The behaviour of these curves allows us to confirm that the propensity rules favour the $\Delta \mathrm{j}$ $= \pm 1$ transitions.

\subsection{Rate coefficients}

The rate coefficients $\left(K_{j \rightarrow j^{\prime}}\right)$, which depend on the kinetic temperature $(T)$, were determined by averaging the cross-sections $\left(\sigma_{j \rightarrow j^{\prime}}\right)$ over the Maxwell-Boltzmann distribution as follows:

$K_{j \rightarrow j^{\prime}}(T)=\left(\frac{8}{\pi \mu \beta}\right)^{1 / 2} \beta^{2} \int_{0}^{\propto} E_{k} \sigma_{j \rightarrow j^{\prime}}\left(E_{k}\right) \mathrm{e}^{-\beta E_{k}} \mathrm{~d} E_{k}$.

Here, $\beta=1 /\left(k_{\mathrm{B}} T\right)$, where $k_{\mathrm{B}}$ is the Boltzmann constant and $\mu$ is the reduced mass (see Table 1). Based on the total energy range, we were able to compute the $\mathrm{AlOH}$ rate coefficients corresponding 
Table 5. Temperature variation of four sets of rate coefficients (in $\mathrm{cm}^{3} \mathrm{~s}^{-1}$ ) for $\Delta j=-1$ transitions.

\begin{tabular}{|c|c|c|c|c|c|c|c|}
\hline System & & Temperature (K) & $1 \rightarrow 0$ & $2 \rightarrow 1$ & $3 \rightarrow 2$ & $4 \rightarrow 3$ & $5 \rightarrow 4$ \\
\hline \multirow[t]{6}{*}{$\mathrm{AlOH}-\mathrm{He}$} & Tchakoua et al. & 10 & $1.079(-9)$ & $7.81(-10)$ & $4.380(-10)$ & $8.152(-10)$ & $2.091(-9)$ \\
\hline & & 30 & $2.087(-10)$ & $1.511(-10)$ & $8.470(-11)$ & $1.576(-10)$ & $4.044(-10)$ \\
\hline & & 150 & $1.870(-11)$ & $1.354(-11)$ & $7.590(-12)$ & $1.413(-11)$ & $3.624(-11)$ \\
\hline & This work & 10 & $7.482(-11)$ & $1.011(-10)$ & $1.098(-10)$ & $1.111(-10)$ & $1.113(-10)$ \\
\hline & & 30 & $6.172(-11)$ & $8.941(-11)$ & $9.756(-11)$ & $1.001(-10)$ & $1.013(-10)$ \\
\hline & & 150 & $5.667(-11)$ & $8.517(-11)$ & $9.326(-11)$ & $9.561(-11)$ & $9.680(-11)$ \\
\hline \multirow[t]{3}{*}{$\mathrm{HCP}-\mathrm{He}^{a}$} & & 20 & $0.796(-11)$ & $1.366(-11)$ & $1.550(-11)$ & $1.343(-11)$ & $1.172(-11)$ \\
\hline & & 50 & $1.072(-11)$ & $1.640(-11)$ & $1.796(-11)$ & $1.758(-11)$ & $1.709(-11)$ \\
\hline & & 150 & $1.748(-11)$ & $2.908(-11)$ & $3.124(-11)$ & $3.182(-11)$ & $3.207(-11)$ \\
\hline \multirow[t]{3}{*}{$\mathrm{AlF}-\mathrm{He}^{b}$} & & 10 & $0.9362(-10)$ & $1.240(-10)$ & $1.396(-10)$ & $1.531(-10)$ & $1.659(-10)$ \\
\hline & & 30 & $1.155(-10)$ & $1.498(-10)$ & $1.630(-10)$ & $1.733(-10)$ & $1.821(-10)$ \\
\hline & & 150 & $1.493(-10)$ & $2.107(-10)$ & $2.262(-10)$ & $2.333(-10)$ & $2.382(-10)$ \\
\hline
\end{tabular}

${ }^{a}$ See Hammami et al. (2008). ${ }^{b}$ See Gotoum et al. (2011).

to the first 13 rotational levels in the kinetic temperature range $5-300 \mathrm{~K}$.

Fig. 6 illustrates the rotational de-excitation rate coefficients for $\Delta j=-1,-2$ and -3 transitions. These curves exhibit that the $\Delta j$ $=-1$ transitions strongly predominate for all kinetic temperatures. Moreover, Table 2 presents the downward rotational rate coefficients computed in this work. These data are very important for the astrophysical community. They allow us to determine the physical conditions of interstellar clouds where $\mathrm{AlOH}$ was observed without assuming the LTE. Even if AlOH mainly collides with $\mathrm{H}_{2}$, which is the most abundant species in the media mentioned above, the $\mathrm{AlOH}-\mathrm{H}_{2}$ rate coefficients can be roughly approximated from those of AlOH-He. As helium is a closed-shell atom with two electrons, the $\mathrm{AlOH}$ rate coefficients induced by collision with para- $\mathrm{H}_{2}(j=$ 0 ) can be derived by multiplying those of $\mathrm{AlOH}-\mathrm{He}$ by the mass scaling factor which is $\sim \sqrt{2}$. Such an estimation was justified in early works on heavy molecules, such as SiS (Lique et al. 2008) and SO (Lique et al. 2007). However, this approximation was not valid for many other systems, including relatively light molecules, (Gotoum et al. 2012; Lanza et al. 2014; Najar et al. 2017). As a result, the present study provides an opportunity to study the collisional de-excitation of $\mathrm{AlOH}$ by $\mathrm{H}_{2}$.

\section{COMPARISON STUDY}

Despite the agreements between our PES and that of Tchakoua et al. (2015), significant differences are noticed in the dynamic results. In fact, our inelastic cross-sections do not exceed $250 \AA^{2}$ while those available in the literature achieve $600 \AA^{2}$. To better appreciate the disagreement, in Table 3 we give both sets of cross-sections computed at $600 \mathrm{~cm}^{-1}$ using different values of $j_{\max }$. For most of the transitions (mainly those for which $\Delta j=1$ ), the old values of cross-sections are 10 to 100 times greater than the new values. We expect that these large values of cross-sections reported by Tchakoua et al. (2015) are due to convergence problems, as they did not take into account the transitions involving $j \geq 10$. The discrepancies mentioned above will persist for the rate coefficients, which are derived by thermally averaging the cross-sections. In Fig. 7, we compare the two sets of rotational rate coefficients by plotting the $5 \rightarrow 4$ and $5 \rightarrow 3$ transitions. As expected, the comparison reveals that the rate coefficients of Tchakoua et al. (2015) are $\sim 100$ times larger than ours, at low kinetic temperatures $(T \leq 50 \mathrm{~K})$. Due to the lack of experimental rate coefficients for $\mathrm{AlOH}-\mathrm{He}$, we rely on the data of HCP-He (Hammami et al. 2008) and AlF-He (Gotoum et al. 2011) in order to settle the matter mentioned above. In fact, these molecules are isoelectronic to $\mathrm{AlOH}$ and $\mathrm{HCP}$ is geometrically identical to it. Moreover, $\mathrm{AlOH}-\mathrm{He}, \mathrm{HCP}-\mathrm{He}$ and $\mathrm{AlF}-\mathrm{He}$ have very similar PESs in term of shape and well depth (see Table 4). All these points support the pertinence of the comparison and predict rate coefficients in the same magnitude rank for the three systems. Therefore, in Table 5 we show the rate coefficients of the three systems for selected temperatures and transitions. The HCP (AlF) data vary from $0.8 \times 10^{-11}$ to $3.3 \times 10^{-11}\left(1.0 \times 10^{-10}\right.$ to $2.4 \times 10^{-10}$ ) and those of $\mathrm{AlOH}$ computed in this work (calculated by Tchakoua et al. 2015) are between $7.5 \times 10^{-11}$ and $1.1 \times 10^{-10}$ $\left(7.6 \times 10^{-12}\right.$ and $\left.1.1 \times 10^{-9}\right)$. According to the comparison made in Table 5, the rate coefficients of $\mathrm{AlOH}$ presented in this paper are more reliable than those provided by Tchakoua et al. (2015).

\section{CONCLUSIONS}

In this paper, we have revisited the rotational excitations and deexcitations of $\mathrm{AlOH}$ due to impact with $\mathrm{He}$. A new two-dimensional PES was computed with the CCSD(T)/aug-cc-pVQZ+bf $a b$ initio method. This PES was then used for the quantum calculations of cross-sections considering total energies up to $1500 \mathrm{~cm}^{-1}$. During the scattering computations, the CC quantum approach was used. By thermally averaging these data over the Maxwell-Boltzmann distribution, rate coefficients of the 13 lowest rotational levels of $\mathrm{AlOH}$ were determined for kinetic temperatures ranging from 5 to $300 \mathrm{~K}$. The new rate coefficients of $\mathrm{AlOH}$ have been compared with the previous ones of Tchakoua et al. (2015) and significant discrepancies are noticed concerning the magnitude ranks. Then we compared both sets of data with those of similar molecules (HCP and AlF). This leads us to conclude that the new rate coefficients of $\mathrm{AlOH}$, which are in good agreement with those of $\mathrm{HCP}$ and $\mathrm{AlF}$, are more reliable than the previous ones. Therefore, we expect that the data presented in this paper will play a key role in the accurate modelling of the abundance of $\mathrm{AlOH}$ in the ISM. In addition, the discrepancies between the previous and new rates of $\mathrm{AlOH}$ mentioned above might encourage experimental work to confirm our findings. 


\section{ACKNOWLEDGEMENTS}

The authors acknowledge Dr François Lique, Laboratoire Ondes et Milieux complexes, UMR CNRS 6294, Université Le Havre, for discussion and advice.

\section{REFERENCES}

Álvarez-Barcia S., Flores J. R., 2016, Phys. Chem. Chem. Phys., 92, 382 Álvarez-Barcia S., Flores J. R., 2011, Chem. Phys., 92, 382

Adlerc T. B., Knizia G., Werner H. J., 2007, J. Chem. Phys., 127, 221106

Apponi A. J., Barclay W. L., Ziurys L. M., 1993, ApJ, 414, 129

Arthurs A. M., Dalgarno A., 1960, Proc. R. Soc. A., 256, 540

Boys S. F., Bernardi F., 1970, Mol. Phys., 19, 553

Cybulski S. M., Toczylowski R., 1999, J. Chem. Phys., 111, 10520

Gotoum N., Nkem C., Hammami K., Charfadine M. A., Owono Owono L. C., Jaidane N., 2011, Ap\&SS, 332, 209

Gotoum N., Nkem C., Hammami K., Owono Owono L. C., Jaidane N., 2012, Ap\&SS, 337, 553

Hammami K., Owono Owono L. C., Jaidane N., Ben Lakhdar Z., 2008, J. Molecular Structure: THEOCHEM, 860, 45

Hauge F. H., Kauffman J. W., Margrave J. L., 1980, J. Am. Chem. Soc., 102, 6005

Hutson J. M., Green S., 1994, MOLSCAT computer code, version 14, Collaborative Computational Project No. 6. Science and Engineering Research Council, UK

Kamiński T. et al., 2016, A\&A, 592, A42

Kendall R. A., Dunning T. H., Harrison R. J., 1992, J. Chem. Phys., 96 , 6796

Knowles P. J., Werner H. J., 1985, Chem. Phys. Lett., 115, 259

Lanza M., Kalugina Y., Wiesenfeld L., Faure A., Lique F., 2014, MNRAS, 443,3351
Li S., Sattelmeyer K. W., Yamaguchi Y., Schaefer H. F., 2003, J. Chem. Phys., 119, 12830

Lique F., Senent M. L., Spielfiedel A., Feautrier N., 2007, J. Chem. Phys., 126, 164312

Lique F., Tobola R., Klos J., Feautrier N., Spielfiedel A., Vincent L. F. M., Chalasiński G., Alexander M. H., 2008, A\&A, 478, 567

McClean R. E., Nelson H. H., Campbell M. L., 1993, J. Phys. Chem., 97, 9673

Manolopoulos D. E., 1986, J. Chem. Phys., 85, 6425

Morton D. C., Drake J. F., Jenkins E. B., Rogerson J. B., Spitzer L., York D. G., 1973, ApJ, 181, 103

Najar F., Naouai M., El Hanini H., Jaidane N., 2017, MNRAS, 472, 2919

Pilgrim J. S., Robbins D. L., Duncan M. A., 1993, Chem. Phys. Lett., 202, 203

Tchakoua T., Pamboundom M., Nsangou M., Motapon O., 2015, Ap\&SS, 357,142

Tenenbaum E. D., Ziurys L. M., 2009, ApJ, 694, 59

Tenenbaum E. D., Ziurys L. M., 2010, ApJ, 712, 93

Tsuji T., 1973, A\&A, 23, 411

Vacek G., Deleeuw B. J., Schaefer H. F., 1993, J. Chem. Phys., 98, 8704

Werner H. J., Knowles P. J., 1985, J. Chem. Phys., 82, 5053

Werner H. J. et al., 2010, MOLPRO,version 2010.1, a package of ab initio programs, http://www.molpro.net

Ziurys L. M., 2006, Proceedings of the National Academy of Sciences, 103 , 12274

Ziurys L. M., Savage C., Highberger J. L., Apponi A. J., Guélin M., Cernicharo J., 2002, ApJ, 564, L45

This paper has been typeset from a $\mathrm{T}_{\mathrm{E}} \mathrm{X} / \mathrm{LT} \mathrm{E} \mathrm{X}$ file prepared by the author. 